\title{
Evaluation of divisor functions of matrices
}

\author{
by \\ Gautami Bhowmik (Nancy)
}

1. Introduction. The study of divisor functions of matrices arose legitimately in the context of arithmetic of matrices, and the question of the number of (possibly weighted) inequivalent factorizations of an integer matrix was asked. However, till now only partial answers were available. Nanda [6] evaluated the case of prime matrices and Narang [7] gave an evaluation for $2 \times 2$ matrices. We obtained a recursion in the size of the matrices and the weights of the divisors $[1,2]$ which helped us obtain a result for $3 \times 3$ matrices but no closed formula for the general case. In this paper we obtain the complete evaluation of the divisor functions by a combinatorial consideration (see Theorem 1). Because of the existence of a bijection (detailed in a forthcoming paper [3]) between the set of divisors of an $r \times r$ integer matrix and the set of subgroups of an abelian group of rank at most $r$, we have here a rather simple proof to obtain the number of subgroups of a finite abelian group.

Acknowledgements. I thank the Mathematisches Institut der Westfälischen Wilhelms-Universität, Münster, Germany, for providing me with the facilities for doing this work.

2. Preliminaries. We consider integer matrices, i.e. matrices with entries from $\mathbb{Z}$. We define factorization of a non-singular $r \times r$ matrix $M$ as

$$
M=M_{1} M_{2},
$$

where taking into account the infinite cardinality of $\mathrm{GL}_{r}(\mathbb{Z})$, we choose $M_{2}$ to be a unique representative of a one-sided equivalence class. Our canonical choice is a matrix in non-singular Hermite Normal Form (HNF), i.e. $M_{2}$ is lower triangular with strictly positive diagonal elements and with every element in the column below the diagonal element belonging to the class modulo the diagonal element. We call $M_{2}$ a divisor of $M$.

1991 Mathematics Subject Classification: 05A15, 11C20, 15A36. 
Associated with inequivalent factorizations of $M$, we define, for any complex number $a$, the divisor function as

$$
\sigma_{a}(M)=\sum_{M=M_{1} M_{2}}\left(\operatorname{det} M_{2}\right)^{a} .
$$

Since $\sigma_{a}(U M V)=\sigma_{a}(M)$ for $U, V$ in $\mathrm{GL}_{r}(\mathbb{Z})$, we choose $M$ to be a unique representative of a two-sided equivalence class, i.e. in Smith Normal Form (SNF). Further, the functions $\sigma_{a}$ are multiplicative, i.e. $\sigma_{a}(A B)=$ $\sigma_{a}(A) \sigma_{a}(B)$ whenever $A$ and $B$ have co-prime determinants. It is, therefore, enough to consider $M$ to be a SNF matrix with prime power determinant. We use the notation $\left\langle f_{1}, f_{2}, \ldots, f_{r}\right\rangle_{p}$ to denote a SNF matrix whose $t$ th diagonal entry is $p^{f_{1}+f_{2}+\ldots+f_{t}}$, where $p$ is a prime number, $f_{1}$ a positive integer and $f_{2}, \ldots, f_{r}$ are non-negative integers. (We may suppress $p$ for notational convenience.) In [1] we proved

\section{LEMMA 1.}

$$
\sigma_{a}\left\langle f_{1}, \ldots, f_{r}\right\rangle_{p}=p^{a} \sigma_{a}\left\langle f_{1}, \ldots, f_{r}-1\right\rangle_{p}+\sigma_{a+1}\left\langle f_{1}, \ldots, f_{r-1}\right\rangle_{p} .
$$

While using the above lemma recursively to evaluate $\sigma$, we encounter the problem that when $f_{r}$ becomes 0 , the SNF structure is destroyed and the recursion cannot be used thereafter without finding the equivalent SNF form. We could not proceed beyond the evaluation of $\sigma_{0}\left\langle f_{1}, f_{2}, f_{3}\right\rangle$ by using it.

3. Evaluations. Now we give a recurrence which preserves the SNF structure. We use the notations $0_{t}$ for a string of $t$ zeros and $\left[\begin{array}{c}k \\ t\end{array}\right]$ for Gaussian polynomials in $p$, defined as

$$
\left[\begin{array}{c}
k \\
t
\end{array}\right]=\frac{\prod_{l=1}^{k}\left(p^{l}-1\right)}{\prod_{m=1}^{t}\left(p^{m}-1\right) \prod_{n=1}^{k-t}\left(p^{n}-1\right)} .
$$

We prove:

Lemma 2. For $k \geq 1$ and $f_{r-k+1} \geq 1$, we have

$$
\begin{aligned}
\sigma_{a} & \left\langle f_{1}, \ldots, f_{r-k+1}, 0_{k-1}\right\rangle_{p} \\
& =\sum_{t=0}^{k-1}\left[\begin{array}{l}
k \\
t
\end{array}\right] p^{a(k-t)} \sigma_{a+t}\left\langle f_{1}, \ldots, f_{r-k+1}-1,0_{k-t-1}\right\rangle_{p}+\sigma_{a+k}\left\langle f_{1}, \ldots, f_{r-k}\right\rangle_{p} .
\end{aligned}
$$

Proof. We construct a tree to descend from $\sigma_{a}\left\langle f_{1}, \ldots, f_{r-k+1}, 0_{k-1}\right\rangle$ to $\sigma_{a+t}\left\langle f_{1}, \ldots, f_{r-k+1}-1,0_{k-t-1}\right\rangle, 0 \leq t<k$. We denote the $b$ th element (from left) of the $c$ th line (from top) by the symbol $\langle\langle c-1, b\rangle\rangle$. This is done by using Lemma 1 repeatedly and realizing that, for $i+1 \leq m \leq k-2$, both

$$
\sigma_{a+i}\left\langle f_{1}, \ldots, f_{r-k+1}-1,0_{m-i}, 1,0_{k-m-2}\right\rangle
$$


and

$$
\sigma_{a+i+1}\left\langle f_{1}, \ldots, f_{r-k+1}-1,0_{m-i-1}, 1,0_{k-m-2}\right\rangle,
$$

i.e. elements $\langle\langle m+1, i+1\rangle\rangle$ and $\langle\langle m+1, i+2\rangle\rangle$ descend to $\langle\langle m+2, i+2\rangle\rangle$, i.e. to

$$
\sigma_{a+i+1}\left\langle f_{1}, \ldots, f_{r-k+1}-1,0_{m-i}, 1,0_{k-m-3}\right\rangle,
$$

the element $\langle\langle m+2, i+2\rangle\rangle$, though in the second case, where we say that a right turn has taken place, there is a multiplication by $p^{a+i+1}$ and in the first case, that of a left turn, such a factor does not exist. In the extreme case where $m=i$, we have $\langle\langle i+1, i+2\rangle\rangle=\sigma_{a+i+1}\left\langle f_{1}, \ldots, f_{r-k+1}, 0_{k-i-2}\right\rangle$. For an example where $r=5$ and $k=4$, see Figure 1 .

We now count the number of ways in which a descent has taken place together with the powers of $p$ that have been attached. We notice that there are exactly $k-t$ right turns to get from $\langle\langle 0,1\rangle\rangle$ to $\langle\langle k, t\rangle\rangle$ and in each such turn the power of $p$ attached is at most $a+t$. We consider the partition function $q(n, u, v)$ that counts the number of ways in which $n$ can be written as the sum of at most $u$ parts, each at most $v$. We remark that the coefficient of $p^{n+a(k-t)}$ that we obtain when we reach $\langle\langle k, t\rangle\rangle$ is precisely $q(n, k-t, t)$. We now use the fact that $\sum p^{n} q(n, k-t, t)=\left[\begin{array}{c}k \\ t\end{array}\right]$.

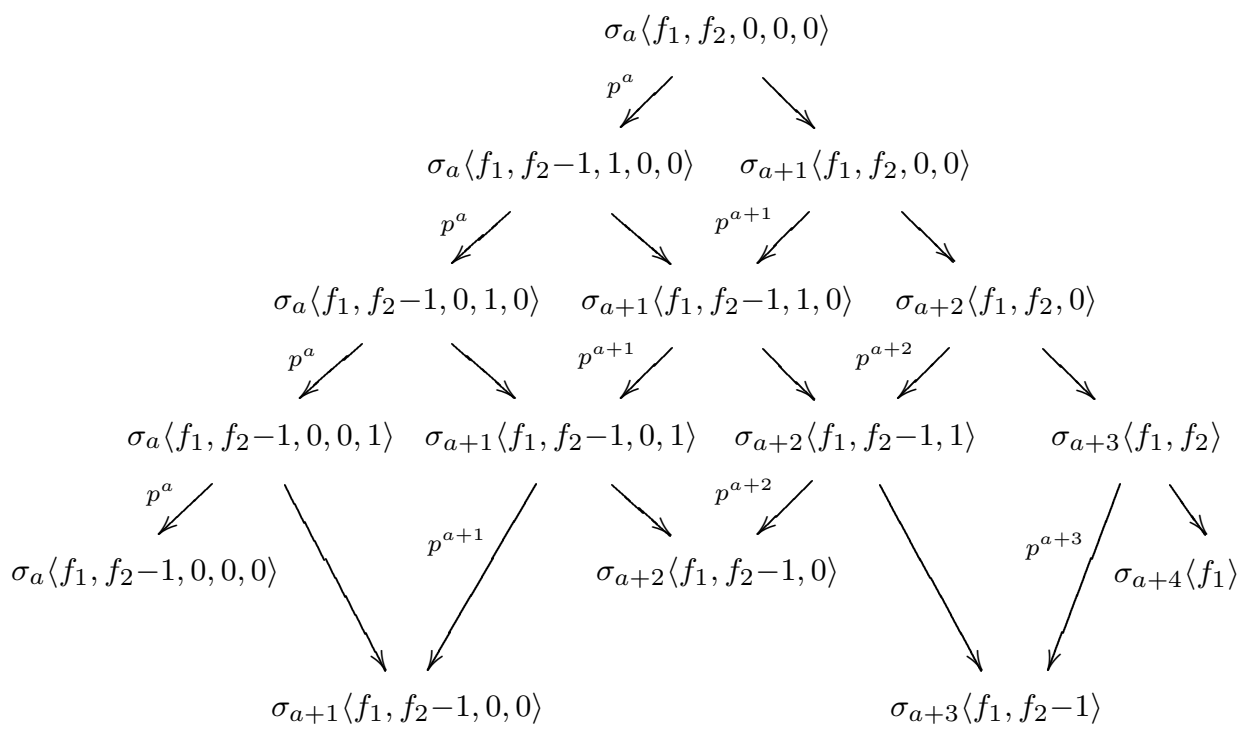

Fig. 1

We now evaluate the divisor functions. We use the notation $\left[\begin{array}{c}k \\ h, \ldots, t\end{array}\right]$ for Gaussian multinomials in $p$, defined as 


$$
\left[\begin{array}{c}
k \\
h, \ldots, t
\end{array}\right]=\frac{\prod_{l=1}^{k}\left(p^{l}-1\right)}{\prod_{i=1}^{h}\left(p^{i}-1\right) \ldots \prod_{m=1}^{t}\left(p^{m}-1\right) \prod_{n=1}^{k-h-\ldots-t}\left(p^{n}-1\right)} .
$$

THEOREM 1.

$$
\begin{array}{r}
\sigma_{a}\left\langle f_{1}, \ldots, f_{r}\right\rangle_{p} \\
=\sum\left[\begin{array}{c}
1 \\
t_{1}, \ldots, t_{f_{r}}
\end{array}\right]\left[\begin{array}{c}
2-t_{1}-\ldots-t_{f_{r}} \\
t_{f_{r}+1}, \ldots, t_{f_{r}+f_{r-1}}
\end{array}\right] \ldots \\
\ldots\left[\begin{array}{c}
r-t_{1}-\ldots-t_{f_{r}+\ldots+f_{2}} \\
t_{f_{r}+\ldots+f_{2}+1}, \ldots, t_{f_{r}+\ldots+f_{1}}
\end{array}\right] p^{\varphi\left(t_{1}, \ldots, t_{f_{r}} ; a\right)}
\end{array}
$$

where the summation is over all $t_{1}, \ldots, t_{f_{r}}$ such that

$$
\left\{\begin{array}{l}
t_{1}+\ldots+t_{f_{r}} \leq 1 \\
t_{1}+\ldots+t_{f_{r}+f_{r-1}} \leq 2 \\
\vdots \\
t_{1}+\ldots+t_{f_{r}+f_{r-1}+\ldots+f_{1}} \leq r
\end{array}\right.
$$

and

$$
\varphi=\sum_{k=1}^{r} \sum_{i=1}^{f_{r-k+1}}\left(k-t_{1}-t_{2}-\ldots-t_{i+s_{k}}\right)\left(a+t_{1}+t_{2}+\ldots+t_{i-1+s_{k}}\right)
$$

with $s_{k}=\sum_{j=1}^{k-1} f_{r-j+1}$. We let $t_{0}$ and all empty sums be 0 and if $f_{r-k+1}=0$, we consider the $k$-th inequality $t_{1}+\ldots+t_{f_{r}+f_{r-1}+\ldots+f_{r-k+1}} \leq k$ to be empty and the $k$-th multinomial to be 1 .

The right hand side of Theorem 1, for $a=0$, counts the total number of subgroups of $\mathbb{Z} / p^{f_{1}} \mathbb{Z} \times \mathbb{Z} / p^{f_{1}+f_{2}} \mathbb{Z} \times \ldots \times \mathbb{Z} / p^{f_{1}+\ldots+f_{r}} \mathbb{Z}[3]$ and extends special cases like the number of subgroups of a given type (see, e.g. [4]) and the number of all subspaces of $(\mathbb{Z} / p \mathbb{Z})^{r}$ (see e.g. [5]).

Proof of Theorem 1 . We use Lemma 2 repeatedly to reduce each non-zero $f_{i}$ to 0 , starting from the right. Let $f_{r}$ be non-zero, i.e. initially $k=1$. By $f_{r}$ iterations of the above lemma, we reduce $f_{r}$ to 0 . This involves summing over $f_{r}$ variables $t_{1}, \ldots, t_{f_{r}}$. We allow each $t_{i}$ to vary from 0 to $1-t_{1}-\ldots-t_{i-1}$, where in the extreme case we get $0_{-1}$ but locally treat it only as a symbol, since at the end of the $f_{r}$ steps the number of zeros will anyway increase by 1 . We then get the product of Gaussian polynomials

$$
\left[\begin{array}{c}
1 \\
t_{1}
\end{array}\right]\left[\begin{array}{c}
1-t_{1} \\
t_{2}
\end{array}\right] \cdots\left[\begin{array}{c}
1-t_{1}-\ldots-t_{f_{r}-1} \\
t_{f_{r}}
\end{array}\right]
$$

which we combine to write the multinomial $\left[\begin{array}{c}1 \\ t_{1}, \ldots, t_{f_{r}}\end{array}\right]$. Now we have to reduce $\sigma_{a+t_{1}+\ldots+t_{f_{r}}}\left\langle f_{1}, \ldots, f_{r-1}, 0_{1-t_{1}-\ldots-t_{f_{r}}}\right\rangle$. If $f_{r}=0$, we have $k=2$ and now that we straight away reduce $f_{r-1}$ to 0 , the first multinomial no 
longer occurs. We follow the same steps recursively to complete the evaluation.

\section{References}

[1] G. Bhowmik, Divisor functions of integer matrices: evaluations, average orders and applications, Astérisque 209 (1992), 169-177.

[2] G. Bhowmik and O. Ramaré, Average orders of multiplicative arithmetical functions of integer matrices, Acta Arith. 66 (1994), 45-62.

[3] - - - Factorisation of matrices, partitions and Hecke algebra, to appear.

[4] L. M. Butler, A unimodality result in the enumeration of subgroups of a finite abelian group, Proc. Amer. Math. Soc. 101 (1987), 771-775.

[5] A. Krieg, Hecke Algebras, Mem. Amer. Math. Soc. 435 (1990).

[6] V. C. Nanda, Arithmetic functions of matrices and polynomial identities, in: Colloq. Math. Soc. János Bolyai 34, North-Holland, 1984, 1107-1126.

[7] A. Narang, Ph. D. Thesis, Panjab University, India, 1979.

DÉPARTEMENT DE MATHÉMATIQUES

UNIVERSITÉ DE NANCY 1

B.P. 239

54506 VANDCEUVRE-LÈS-NANCY, FRANCE

E-mail: BHOWMIK@IECN.U-NANCY.FR 